\title{
Une nouvelle approche pour l'optimisation fiabiliste
}

\section{A new approach for the Reliability Based Design Optimization}

\section{Fatma ABID ${ }^{1}$, Abdelkhalak ELHAMI ${ }^{2}$, Tarek MERZOUKI ${ }^{3}$, Lassaad WALHA ${ }^{4}$, Mohamed HADDAR $^{5}$}

\author{
${ }^{1}$ Laboratoire de mécanique de Normandie, Institut national des sciences appliquées de Rouen, Rouen, France, \\ fatma.abid@insa-rouen.fr \\ ${ }^{2}$ Laboratoire de mécanique de Normandie, Institut national des sciences appliquées de Rouen, Rouen, France, \\ Abdelkhalak.elhami@insa-rouen.fr \\ ${ }^{3}$ Laboratoire Ingénierie des Systèmes de Versailles, Université de Versailles Saint Quentin en Yvelines, Velizy, \\ France, tarek.merzouki@uvsq.fr \\ ${ }^{4}$ Laboratoire de Mécanique, Modélisation et Productique, Ecole national d'ingénieurs de Sfax, Sfax, Tunisie, \\ walhalassaad@yahoo.fr \\ ${ }^{5}$ Laboratoire de Mécanique, Modélisation et Productique, Ecole national d'ingénieurs de Sfax, Sfax, Tunisie, \\ mohamed.haddar@enis.rnu.tn
}

RÉSUMÉ. Le but de cet article est de présenter une nouvelle méthode d'optimisation fiabiliste (RBDO). Celle-ci est formé par des facteurs de sécurité dérivés de la méthode des facteurs optimaux de sureté (OSF) qui est couplée à l'optimisation des essaims particulaires (PSO). Une telle méthode élimine l'utilisation de l'analyse de fiabilité dans RBDO. L'objectif de ce processus d'optimisation est de réduire le temps de calcul comparé au processus RBDO classique. L'étude illustrative présentée dans cet article montre que le couplage de la méthode des facteurs optimaux de sureté avec un algorithme évolutif effectue le RBDO d'un modèle mathématique.

ABSTRACT. The purpose of this paper is to present a new Reliability Based Design Optimization (RBDO) method. This one consists in safety factors derived from Optimum Safety Factors (OSF) method which is coupled with Particle Swarm Optimization (PSO). Such method eliminates the employ of reliability analysis in RBDO. The objective of this optimization process is to reduce the computational time comparing with classical RBDO process. The illustrative study presented in this paper shows that the coupling of Optimum Safety Factors method with evolutionary algorithm performs the RBDO of a mathematical model.

MOTS-CLÉS. Optimisation fiabiliste (RBDO), Optimisation des essaims particulaires (PSO), facteurs optimaux de sureté (OSF). KEYWORDS. Reliability Based Design Optimization (RBDO), Particle Swarm Optimization (PSO), Optimum Safety Factors (OSF).

\section{Introduction}

Nowadays, researchers try to minimize the cost of a mechanical system taking into consideration a given level of reliability. The aim of the Reliability Based Design Optimization (RBDO) is to design structures that should be reliable and economical.

In deterministic design optimization, the uncertainties of the system parameters are not taken into account. As a result, the optimal design obtained does not ensure the target reliability level. In fact, the resulting optimum solution may lead to a high risk of failure. Thus, Reliability Based Design Optimization (RBDO) method is applied to provide an enhanced design with high level of confidence. The main RBDO formulations can be classified into three categories namely two level approach, decoupled approach and single loop approach.

At first, the two level RBDO approach considers the reliability assessment inside the optimization loop. In fact, the outer loop of this approach deals with optimization while the inner loop deals with reliability evaluation. The main drawback of this approach is the numerical effort required to solve the system. Alternatively, others articles are concentrated on developing efficient RBDO formulations based on either single loop technique or decoupled reliability analysis. These methods aim at avoiding the reliability analysis in the optimizing loop by introducing equivalent optimality conditions as an alternative of the reliability constraints themselves. The single loop approach consists in solving the RBDO problems in a single loop process, where the reliability analysis is avoided. This approach is 
summarized in [DU 04], [YAN 04], and [YAN 05]. The decoupled approach consists in separating the optimization procedure from the reliability analysis. Examples of this method is cited in [KUS 00], [CHE 06], [AGA 07], [Yi 08a], [Yi 08b].

This work is structured as follows: The RBDO model is presented next in section 2. One of the main contributions of this work, the new RBDO methodology is fully presented in section 3 . The PSO method is briefly described in section 4 . The safety factor method is demonstrated on classical studies in section 5. Finally, comments about the extension of the methodology to more complex structures and its limitations are in section 6.

\section{RBDO model}

The basic RBDO formulation consists in minimizing the objective function under probabilistic constraints. In RBDO, two types of variables are considered: design variables $x$ and random variables $y$. Typical RBDO formulation is written as [AOU 10]:

$$
\begin{aligned}
& \min _{x}: f(x) \\
& \text { subject to }\left(\begin{array}{l}
\operatorname{Pr}\left[G_{i}(x, y) \leq 0\right] \leq P_{f_{i}}^{T} \quad i=1, \ldots, m \\
h_{j}(x) \leq 0 \quad j=m+1, . ., M
\end{array}\right.
\end{aligned}
$$

Where $x$ is the vector of design variables, $y$ is the vector of random variables, $f$ is the objective function, $G_{i}$ is the performance function, $h_{j}$ are deterministic constraints, $\operatorname{Pr}[$.$] is the probability$ operator, $P_{f_{i}}^{T}$ is the admissible failure probability, $m$ is the number of performance functions and $M$ is the total number of constraints.

It is to be noted that the design variables $x$ can be either independent deterministic variables or probability distribution parameters. The deterministic constraints $h_{j}$ are the upper and lower bounds of the design variables. The failure probability is given by:

$$
\operatorname{Pr}\left[G_{i}(x, y) \leq 0\right]=\int \ldots \int_{G_{i}(x, y) \leq 0} f_{y}(y) d y
$$

Where $f_{y}(y)$ is the joint density function of random variables $y$.

\subsection{Two level RBDO approach}

The direct solution of RBDO problem is formed by a two level approach where the outer loop aims at solving the optimization problem in terms of design variables $\mathrm{d}$ and the inner loop aims at solving the reliability problem in terms of random variables $x$.

In order to reduce computation effort, two main formulations have been proposed to deal with probabilistic constraint:

-The Reliability Index Approach (RIA) consists in using FORM approximation to achieve the reliability analysis. In this case, the reliability index constraint replace the probabilistic constraints.

-The Performance Measure Approach (PMA) involves an inverse reliability problem where the probabilistic constraints are transformed to performance measures which correspond to the target reliability level. 


\section{Proposed RBDO methodology and its coupling with global algorithm}

As the goal is the couple reliability analysis with global optimization algorithms, a new RBDO methodology that differs from the ones described in section 2 is proposed here. The main idea is to estimate the Most Probable Point (MPP) of an optimal design (approximate $u^{*}$ or $x^{*}$ of $m^{\text {optimal }}$ ) obtained using any global optimization algorithm. After that, we calculate the safety factors $S_{f}$ applied to such points in order to obtaining the final design $m^{\text {reliable }}$ that guarantees the prescribed reliability level of the structure. Such a methodology is described in the following.

First, the safety factors are deduced from the Optimum safety factors (OSF) which is given by Kharmanda [KHA 04], [KHA 07], [KHA 14] .

In order to calculate the RBDO of a given system, the maximum probability of failure of such a system or its reliability index $\beta^{\text {target }}$ is defined. At the end of the RBDO process, such reliability index must be achieved. Therefore, the optimum must verify $\left\|u^{*}\left(m^{\text {reliable }}\right)\right\|=\beta^{\text {target }}$.

As a result, $u^{*}\left(m^{\text {reliable }}\right)$ is written as follows:

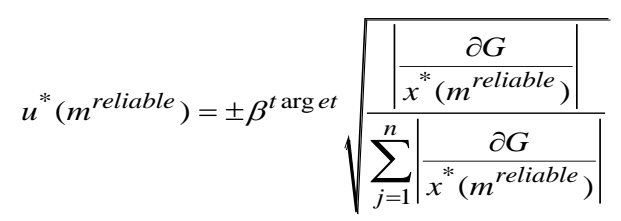

Where the \pm sign depends on the sign of the derivative:

$$
\left|\frac{\partial G}{x^{*}\left(m^{\text {reliable }}\right)}\right|>0 \rightarrow u^{*}\left(m^{\text {reliable }}\right)>0
$$

and

$$
\left|\frac{\partial G}{x^{*}\left(m^{\text {reliable }}\right)}\right|<0 \rightarrow u^{*}\left(m^{\text {reliable }}\right)<0
$$

Furthermore, in general, for a normal distribution of the random variable, we consider the transformation to the normalized space ( $\mathrm{u}$ space) for the physical space ( $\mathrm{x}$ space) as defined in equation:

$$
u_{i}=\frac{x_{i}-m_{x_{i}}}{\sigma_{i}} \quad i=1, . ., n
$$

The standard deviation $\sigma_{i}$ can be related to mean value $m_{x_{i}}$ by:

$$
\sigma_{i}=\gamma_{i} . m_{x_{i}} \quad i=1, \ldots, n
$$

This way, we introduce the safety factors $S_{f_{i}}$ corresponding to the design variable $x_{i}$, the design point can be expressed by:

$$
m_{x_{i}}=S_{f_{i}} \cdot x_{i}
$$

The equation of the optimum safety factor for a single limit state can be written in the following form:

$$
S_{f_{i}}=1 \pm \gamma_{i} \cdot u^{*}\left(m^{\text {reliable }}\right)
$$


Here, the sign \pm depends on the sign of the derivative as follows :

$$
\frac{\partial G}{\partial x_{i}}>0 \rightarrow S_{f_{i}}>0
$$

and

$$
\frac{\partial G}{\partial x_{i}}<0 \rightarrow S_{f_{i}}<0
$$

Where $S_{f_{i}}$ is the safety factor of the $\mathrm{i}$ th design variable ( $\mathrm{i}=1, . ., \mathrm{Np}$ ) . Considering uncertainties only on the design variables of the problem and assuming that the problem has only one constraint, we can conclude that the vector $x^{*}\left(m^{\text {reliable }}\right)$ is estimated by solving the deterministic optimization of the problem. In others words, we can notice that $x^{*}\left(m^{\text {reliable }}\right) \approx x^{*}\left(m^{\text {optimal }}\right)$. Then, applying the safety factors to the vector $x^{*}\left(m^{\text {optimal }}\right)$, the result of the RBDO problem $m^{\text {reliable }}$ is obtained guaranteeing the target reliability of the structure. As shown in the derivation of safety factors, the methodology described does not make the reliability analysis. Thus, reducing the computational cost of the RBDO.

The main steps of the implementation of the methodology are as follows:

Step 1: Estimate the MPP on the physical space of the optimal design $m^{\text {optimal }}$ by solving the deterministic optimization of the problem. Any deterministic optimization algorithm may be employed to obtain $x^{*}\left(m^{\text {optimal }}\right)$ making it possible to couple the RBDO methodology with any global optimization algorithm. In this work, PSO is used to effect this step.

Step 2: After a sensitivity analysis on $x^{*}\left(m^{\text {optimal }}\right)$, compute the safety factor $S_{f}$.

Step 3: Calculate the optimal solution, the safety factors are applied to the vector $x^{*}\left(m^{\text {optimal }}\right)$ finding the final design $m^{\text {reliable }}$ that guarantees the minimum allowed reliability level of structure.

As mentioned before, the main advantage of this method would be that the computational effort is really close to the standard deterministic optimization. However, the methodology possess some limitations such as:

- Only the design variable can be treated as random variables.

- In the form the methodology was presented, it is limited to one active probabilistic constraint.

\section{Particle swarm optimization}

Particle Swarm Optimization (PSO) is one of the most popular nature-inspired metaheuristic optimization algorithm. Recently, PSO is used in solving various optimization problems in the field of science and engineering [ALA 16].

In particle swarm algorithm, each particle $\mathrm{i}$ is treated as point in a space with dimensions $\mathrm{d}$, a position $X_{i}$, a velocity $V_{i}$ and a personal best position Xbest $_{i}$. The personal best position associated with a particles in the swarm is represented by the vector Xgbest. After finding the best values, the particle updates its velocity and positions with the following equations [ELL 13]:

$$
V_{i}(t+1)=w V_{i}(t)+\rho_{1} \text {.rand }(1) \cdot\left[X_{\text {best }}(t)-X_{i}(t)\right]+\rho_{2} \text {.rand }(2) \cdot\left[X g b e s t(t)-X_{i}(t)\right]
$$

and

$$
X_{i}(t+1)=X_{i}(t)+V_{i}(t+1)
$$


$\rho_{1}$ and $\rho_{2}$ are the balance factors between the effect of self knowledge and social knowledge in moving the particle towards the target. $\operatorname{rand}(1)$ and $\operatorname{rand}(2)$ are independent random number in the rang $[0,1]$.

\section{Mathematical model}

The RBDO methods presented in the previous sections are applied to solve analytical non linear function. The problem contains three designs variables $\left\{d_{1}, d_{2}, d_{3}\right\}$ and three random variables $\left\{x_{1}, x_{2}, x_{3}\right\}$ which are normally distributed with respective means $\{5,5,5\}$.

The coefficient of variations are equal to 0.5 for each variables. The target reliability level $\left(\beta_{t}=2,3,4\right)$. Three initial points are tested: $d_{1}^{0}, d_{2}^{0}, d_{3}^{0}$.

$$
\begin{aligned}
& \min _{\mathrm{d}}: 10 \cdot\left(\mathrm{d}_{1}-1\right)^{2}+20 \cdot\left(\mathrm{d}_{2}-1\right)^{2}+30 \cdot\left(\mathrm{d}_{3}-1\right)^{2} \\
& \text { subject to : } \\
& \qquad \begin{array}{l}
\operatorname{Pr}\left[\left(\mathrm{x}_{1}^{2}+2 \mathrm{x}_{2}-\mathrm{x}_{3}\right) \leq \mathrm{O}\right] \leq \mathrm{P}_{\mathrm{f}}^{\mathrm{T}} \\
\mathrm{O} \leq \mathrm{d}_{1} \leq 10 \\
O \leq \mathrm{d}_{2} \leq 10 \\
\\
O \leq \mathrm{d}_{3} \leq 10
\end{array}
\end{aligned}
$$

\begin{tabular}{|c|c|c|c|c|c|c|c|c|c|}
\hline \multirow[b]{2}{*}{$\boldsymbol{\beta}=\mathbf{3}$} & \multicolumn{3}{|c|}{ Safety factor } & \multicolumn{3}{|c|}{ RBDO RIA } & \multicolumn{3}{|c|}{ RBDO PMA } \\
\hline & $\mathbf{d}_{\mathbf{1}}$ & $\mathbf{d}_{2}$ & $\mathbf{d}_{\mathbf{3}}$ & $\mathbf{d}_{\mathbf{1}}$ & $\mathbf{d}_{\mathbf{2}}$ & $\mathbf{d}_{3}$ & $\mathbf{d}_{1}$ & $\mathbf{d}_{\mathbf{2}}$ & $\mathbf{d}_{\mathbf{3}}$ \\
\hline$m^{\text {reliable }}$ & 0.5725 & 2.0203 & 2.4740 & 1.7183 & 2.4345 & 2.8551 & 1.7183 & 2.4345 & 2.8552 \\
\hline Objective function & \multicolumn{3}{|c|}{10.1358} & \multicolumn{3}{|c|}{9.5647} & \multicolumn{3}{|c|}{9.5647} \\
\hline Time (s) & \multicolumn{3}{|c|}{0.6961} & \multicolumn{3}{|c|}{9.7787} & \multicolumn{3}{|c|}{10.0311} \\
\hline
\end{tabular}

\begin{tabular}{|c|c|c|c|c|c|c|c|c|c|c|}
\hline & & \multicolumn{3}{|c|}{ Safety factor } & \multicolumn{3}{|c|}{ RBDO RIA } & \multicolumn{3}{|c|}{ RBDO PMA } \\
\hline & & $d_{1}$ & $d_{2}$ & $\mathbf{d}_{\mathbf{3}}$ & $d_{1}$ & $\mathbf{d}_{2}$ & $\mathbf{d}_{\mathbf{3}}$ & $d_{1}$ & $d_{2}$ & $\mathbf{d}_{\mathbf{3}}$ \\
\hline \multirow{3}{*}{$\boldsymbol{\beta}=\mathbf{2}$} & $\mathbf{m}^{\text {reliable }}$ & 0.5370 & 1.8510 & 2.7308 & 1.2384 & 2.1691 & 2.9437 & 1.2384 & 2.1690 & 2.9436 \\
\hline & $\begin{array}{l}\text { Objective } \\
\text { function }\end{array}$ & \multicolumn{3}{|c|}{4.7625} & \multicolumn{3}{|c|}{1.2349} & \multicolumn{3}{|c|}{1.2349} \\
\hline & Time (s) & \multicolumn{3}{|c|}{0.6906} & \multicolumn{3}{|c|}{3.8821} & \multicolumn{3}{|c|}{3.0404} \\
\hline \multirow{3}{*}{$\beta=4$} & $\mathrm{~m}^{\text {reliable }}$ & 0.6211 & 2.2511 & 2.1238 & 2.2345 & 2.6537 & 2. 7820 & 2.2347 & 2.6536 & 2.7821 \\
\hline & $\begin{array}{l}\text { Objective } \\
\text { function }\end{array}$ & \multicolumn{3}{|c|}{25.7270} & \multicolumn{3}{|c|}{25.2127} & \multicolumn{3}{|c|}{25.2127} \\
\hline & Time (s) & \multicolumn{3}{|c|}{0.2316} & \multicolumn{3}{|c|}{3.3620} & \multicolumn{3}{|c|}{5.9610} \\
\hline
\end{tabular}

Tableau 1. RBDO results of non linear limit state

Tableau 2. Influence of $\beta$ on computational time

The linear non limit state has been evaluated for different values of target reliability index $\beta^{\text {target }}$. The result show that the computational cost of the proposed method does not depend of the reliability 
target. Classical strategies may have their costs seriously impacted. However, the safety factor method always gives a higher final design area.

\section{Conclusion}

In this work, a new safety factors based on RBDO methodology was employed and validated in the optimization of mathematical model. The following conclusions can be drawn from the analysis performed in this work: (1) The coupling of the PSO with the proposed safety factors methods may performed the RBDO of mathematical example. (2) Such a method is able to perform the RBDO of structure with only extra computational effort than deterministic optimization being suitable for coupling with global optimization algorithms. Although the illustrative model used in the numerical analysis of this article is simple, the RBDO methodology can be directly extended to more complex examples using for instance the finite element method. However, the method is limited because only the design variables can be treated as random variables and one active probabilistic constraint can be taken into account. To overcome such limitations, further research is needed.

\section{References}

[AGA 07] AGARWAL H., MOZUMDER C. K., RENAUD, J. E., WATSON L. T., «An inverse-measure-based unilevel architecture for reliability-based design optimization», Structural and Multidisciplinary Optimization, vol. 33, n. 3, p. 217-227, 2007.

[ALA 16] ALAM, M. N., «Particle Swarm Optimization: Algorithm and its Codes in MATLAB», 2016.

[ANT 06] ANTONIO C. C., «A hierarchical genetic algorithm with age structure for multimodal optimal design of hybrid composites», Structural and Multidisciplinary Optimization, vol. 31, n. 4, p. 280-294, 2006.

[AOU 10] AOUES Y., CHATEAUNEUF A., «Benchmark study of numerical methods for reliability-based design optimization». Structural and multidisciplinary optimization, vol. 41, n. 2, p. 277-294, 2010.

[CHE 06] CHENG G.D., XU L., AND JIANG L., «Sequential approximate programming strategy for reliability-based optimization», Computer and Structures, vol. 84, n. 21, p. 1353-1367, 2006.

[DU 04] DU X., CHEN W., «Sequential optimization and reliability assessment method for efficient probabilistic design», Transactions-American Society of Mechanical Engineers Journal of Mechanical Design, p. 225-233, 2004.

[ELl 13] EllaiA, R., HABBAL, A., PAGNACCO, E., «A New Accelerated Multi-objective Particle Swarm Algorithm», Applications to Truss Topology Optimization. In 10th World Congress on Structural and Multidisciplinary Optimization, May 2013.

[KHA 14] KHARMANDA G., IBRAHIM M. H., AL-KHEER A. A., GUERIN F., EL-HAMI A., «Reliability-based design optimization of shank chisel plough using optimum safety factor strategy», Computers and electronics in agriculture, vol. 109, p. 162-171, 2014.

[KHA 04] KHARMANDA, G., OLHOFF, N., EL-HAMI, A., «Optimum values of structural safety factors for a predefined reliability level with extension to multiple limit states», Structural and Multidisciplinary Optimization, vol. 27, n. 6, p. 421-434, 2004.

[KHA 07] KHARMANDA, G., OLHOFF, N., «Extension of optimum safety factor method to nonlinear reliability-based design optimization», Structural and Multidisciplinary Optimization, vol. 34, n. 5, p. 367-380, 2007.

[KEN 95] KENNEDY J., EBERHART R., Proceedings of IEEE international conference on neural networks, Perth, Australia, 1995

[KUS 00] KUSCHEL N., RACKWITZ R., «A new approach for structural optimization of series systems», Applications of Statistics and Probability, vol. 2, n. 8, p. 987-994, 2000.

[YAN 04] YANG R. J., GU L., «Experience with approximate reliability-based optimization methods», Structural and Multidisciplinary Optimization, vol. 26, p. 152-159, 2004.

[YAN 05] YANG R. J., CHUANG C., GU L., LI, G., «Experience with approximate reliability-based optimization methods II: an exhaust system problem », Structural and Multidisciplinary Optimization, vol. 29, n. 6, p. 488-497, 2005 . 
[YI 08a] YI P., CHENG G., «Further study on efficiency of sequential approximate programming for probabilistic structural design optimization», Structural and Multidisciplinary Optimization, vol. 35, n. 6, p. 509-522, 2008.

[YI 08b] YI P., CHENG G., JIANG L., «A sequential approximate programming strategy for performance-measurebased probabilistic structural design optimization». Structural Safety, vol. 30, n. 2, p. 91-109, 2008. 